\title{
Survey radiography and computerized tomography imaging of the thorax in female dogs with mammary tumors
}

\author{
Carolina C Otoni ${ }^{*}$, Sheila C Rahal ${ }^{1}$, Luiz C Vulcano², Sérgio M Ribeiro ${ }^{3}$, Khadije Hette ${ }^{1}$, Tatiana Giordano ${ }^{\text {, }}$ \\ Danuta P Doiche ${ }^{2}$, Renée L Amorim ${ }^{4}$
}

\begin{abstract}
Background: Accurate early diagnosis of lung metastases is important for establishing therapeutic measures. Therefore, the present study aimed to compare survey thoracic radiographs and computerized tomography (CT) scans to specifically identify lung metastases in female dogs with mammary tumors.

Methods: Twenty-one female dogs, weighing 3 to $34 \mathrm{~kg}$ and aged from 5 years to 14 years and 10 months, with mammary tumors were studied. In all dogs before the imaging examinations, fine-needle aspiration cytology of the mammary tumors was performed to confirm the diagnosis. Three-view thoracic radiographs were accomplished: right lateral, left lateral and ventrodorsal views. Sequential transverse images of the thorax were acquired on a spiral Scanner, before and after intravenous bolus injection of nonionic iodine contrast. Soft-tissue and lung windows were applied. All the mammary tumors were surgically removed and examined histologically.

Results: The correlation between the cytological and histological results regarding presence of malignancy was observed in only 17 cases. In radiographic examinations, no dog displayed signs of lung metastases or thorax chest lesions. CT detected lung metastasis in two cases, while small areas of lung atelectasis located peripherally were found in $28.57 \%$ of the dogs.
\end{abstract}

Conclusion: In this study population, spiral CT showed higher sensitivity than chest radiographies to detect lung metastasis; this indicates that $\mathrm{CT}$ should be performed on all female dogs with malignant mammary tumors.

\section{Background}

Mammary tumors constitute the most frequent neoplastic disease in female dogs [1,2]. The disease etiology has not yet been totally elucidated, but there are indications of a hormonal dependence because the incidence of tumors is reduced by using early ovariohysterectomy, with better results when the procedure is performed before the first estrus [1-3]. In addition, dogs with mammary gland carcinoma spayed less than 2 years before tumor surgery live longer than dogs spayed earlier in relation to such surgery [4].

Approximately $35 \%$ to $50 \%$ of all mammary tumors in female dogs are considered malignant by histological

\footnotetext{
* Correspondence: carolotonivet@yahoo.com.br

'São Paulo State University (Unesp), Department of Veterinary Surgery and Anesthesiology, School of Veterinary Medicine and Animal Science, Botucatu, SP, Brazil
}

(c) 2010 Otoni et al; licensee BioMed Central Ltd. This is an Open Access article distributed under the terms of the Creative Commons Attribution License (http://creativecommons.org/licenses/by/2.0), which permits unrestricted use, distribution, and reproduction in any medium, provided the original work is properly cited. examinations [2,3]. The clinical stage, tumor size and ovariohysterectomy status are prognostic factors for dog survival after surgery to treat malignant mammary tumors [5]. Furthermore, these tumors may disseminate through the lymphatic and blood vessel routes to the regional lymph nodes and lungs $[1,3]$. Therefore, the accurate and early diagnosis of lung metastases is of considerable importance in the establishment of therapeutic measures, and approximately $25 \%$ and $50 \%$ of the female dogs with malignant mammary tumors already present them at the moment of the physical examination [3].

Among the imaging methods reported as frequently used for lung metastases identification are radiographic examinations, magnetic resonance and computerized tomography (CT) [3,6-9]. Radiographically, lung metastasis can be characterized by well-defined nodules, 
poorly demarcated nodules or as pleural effusion without any evidence of lung lesions [2]. Although CT is considered a more sensitive method than radiography for detecting lung metastases, false-positive or falsenegative results may occur $[8,10,11]$. Therefore, various types of CT instruments are constantly being developed to obtain higher accuracy and potency. Furthermore, a short exposure time is very important to minimize the effects of cardiovascular and respiratory motion [12].

The present study aimed to compare survey thoracic radiographs and computerized tomography (CT) scans in relation to their ability to specifically identify lung metastases in female dogs with mammary tumors.

\section{Materials and methods}

Twenty-one female dogs, weighing 3 to $34 \mathrm{~kg}$ and aged from 5 years to 14 years and 10 months (average of 9 years old and 5 months), with mammary tumors were utilized (Table 1). The time between tumor identification and surgical removal varied from 2 months to 1 year. Two dogs (Cases 6 and 19) had been submitted 3 months previously for regional mastectomy based on the lymphatic drainage in the other mammary chain. Four female dogs (Cases 1, 3, 6 and 21) had already been spayed. Before the imaging examinations in all female dogs, fine-needle aspiration cytology of the mammary tumors was performed to confirm the diagnosis. Only those with malignant mammary tumors were included in the study. The size of the primary tumor was classified according to maximum diameter as follows: $\mathrm{T} 1<3 \mathrm{~cm}$, T2 $3-5 \mathrm{~cm}$ and $\mathrm{T} 3>5 \mathrm{~cm}$ (Table 1 ). Laboratory tests, including a complete blood cell, urinalysis and chemistry panel, were carried out to detect any metabolic alterations that could contraindicate the procedures.

\section{Preoperative Radiographic and CT studies}

Thoracic radiographs were taken from three views: right lateral, left lateral and ventrodorsal. Green-sensitive film (Kodak) and cassettes with screens were used. A focusfilm distance of $90 \mathrm{~cm}$ was used with an exposure of $50-70 \mathrm{kV}$ and 3.2-5.0 mAs for the lateral view and 45$65 \mathrm{kV}$ and 3.2-5.0 $\mathrm{mAs}$ for the ventrodorsal view, according to the size of each dog. The radiographs were done at peak inspiration. An X-ray unit type TUR D800-4, $125 \mathrm{kVp} / 500 \mathrm{~mA}$ capacity, equipped with Potter-Bucky grid was used. The film was processed with an automatic processor (Macrotec MX2). To maintain the quality control of the radiographs, in both ventrodorsal and lateral views the thorax should not be rotated. In addition, the sternum and vertebra should be superimposed on the ventrodorsal view, and little or no contact between the diaphragm and the heart should be seen on the lateral view. The technical quality of the film was considered satisfactory if there was a clear contrast among the structures - pulmonary vessels, heart and air-filled lungs. The thoracic radiographs were evaluated starting from cardiac silhouette, trachea, mediastinum and pleural space, and ending at the lungs. Radiographs were classified as either positive or negative for pulmonary metastases, and other pulmonary diseases. In addition, the heart aspect, the presence of mediastinal masses, and thoracic wall alteration were evaluated.

To perform CT examinations the female dogs were premedicated with acepromazine $0.03 \mathrm{mg} / \mathrm{kg}$, IM, and morphine $0.5 \mathrm{mg} / \mathrm{kg}$, IM. After approximately $10 \mathrm{~min}$ utes, dissociative anesthesia was induced and maintained with ketamine $3 \mathrm{mg} / \mathrm{kg}$ and diazepam $0.5 \mathrm{mg} / \mathrm{kg}$, administered intravenously, with the dogs positioned in sternal recumbency. The lungs were not mechanically inflated for imaging. The female dogs were placed in dorsal recumbency with the forelimbs pulled cranially and the hind limbs caudally. Sequential transverse images from the first thoracic vertebra to lumbar diaphragmatic recess were acquired on a spiral Scanner (Shimadzu SCT-7800CT). After plain CT study, a contrast study was done using $2 \mathrm{ml} / \mathrm{kg}$, IV, bolus injection of nonionic iodine contrast agent (iohexol or meglumine diatrizoate). The scanning parameters were $120 \mathrm{kVp}$, 150-180 mA, 2.0-3.0 mm collimation, 2:0 pitch, and 1second scan time. The field of view was $168-409 \mathrm{~mm}$ according to the dog's size, while the matrix was $512 \times$ $512 \mathrm{~mm}$. The software used to read the CT-images was 2.1.2 eFilm (TM) Lite (TM) (MERGE Healthcare). The study was carried out using soft-tissue and lung windows, and it was started from the mediastinum to chest wall in order to detect lesions. The plain CT images were compared with the contrast images. CT images were classified as either positive or negative for pulmonary metastases. When nodules were present, their diameters were found by means of a measuring tool from an image analysis software package. Three experienced radiologists performed blind evaluation of the radiographs and CT.

\section{Surgical procedures, and Histology}

After ovariohysterectomy, either a unilateral, bilateral or regional mastectomy was performed, based on an assessment of lymphatic drainage. Lumpectomy was used specifically in cases when other benign masses were present.

The mammary tumors removed at surgery were immediately fixed in 10\% buffered formalin. Semi-serial sections, $4-\mu \mathrm{m}$-thick, were obtained and stained with hematoxylin and eosin. The histological results were compared with those obtained previously by fine-needle aspiration cytology. 


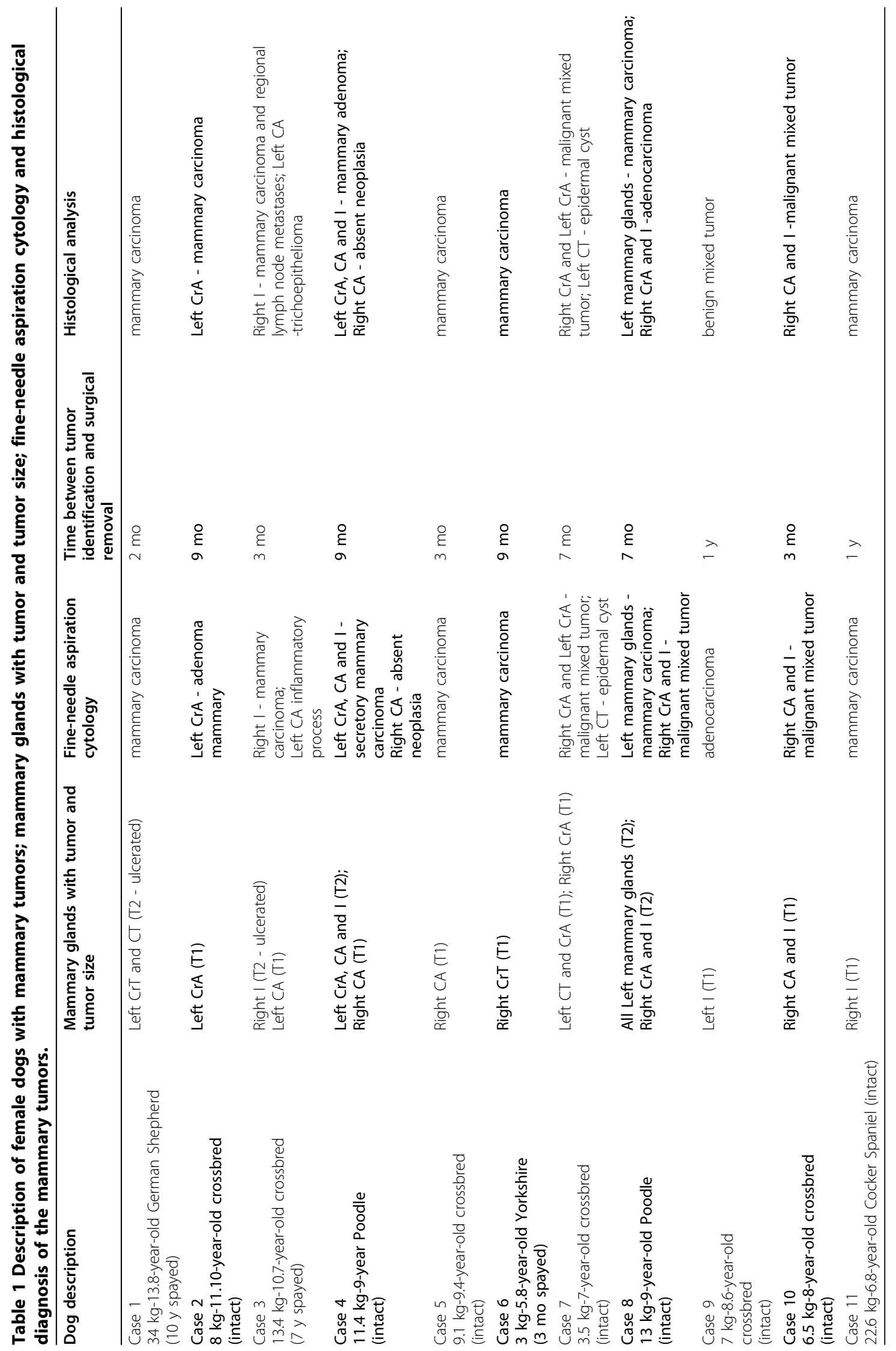




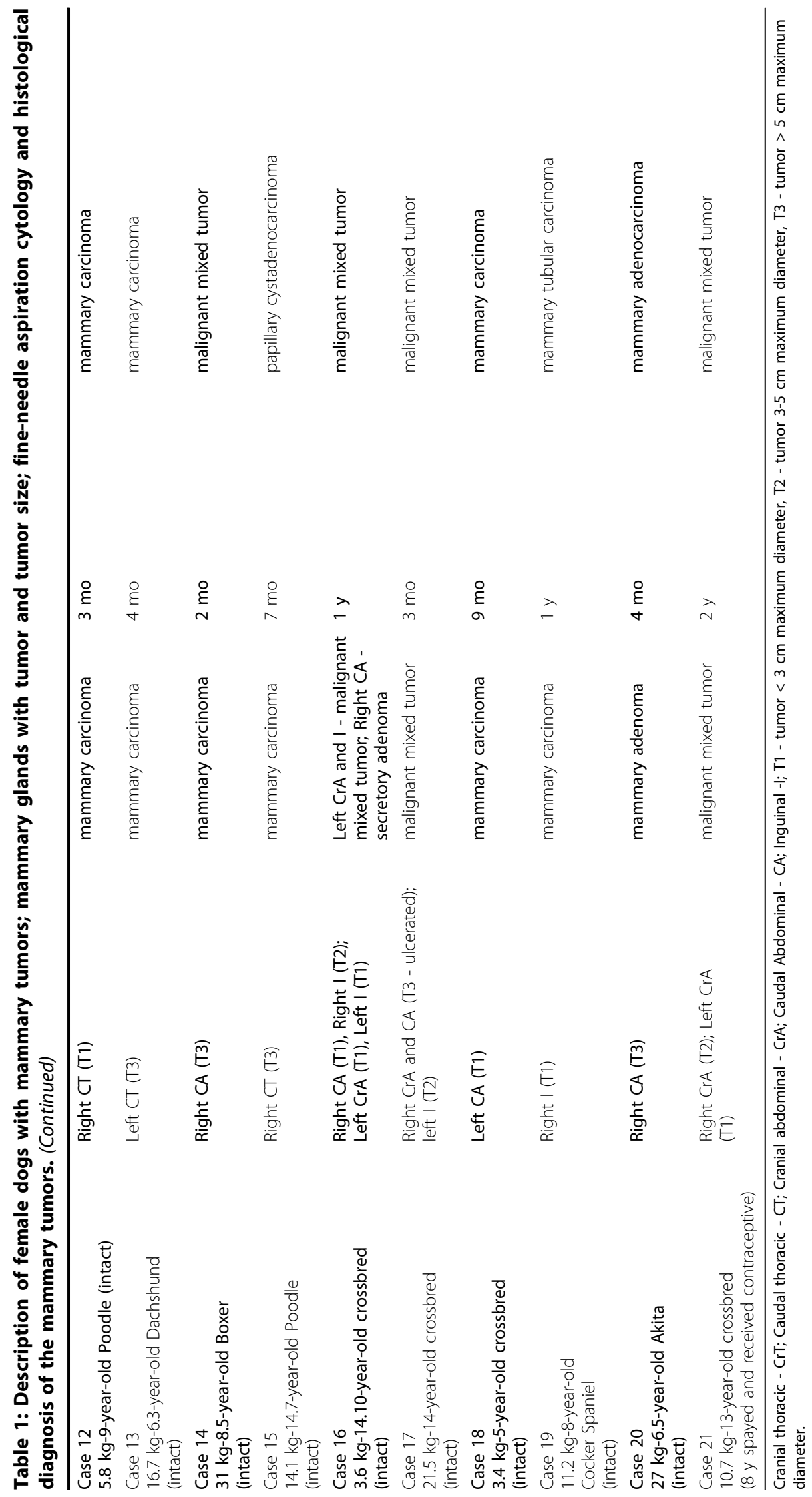




\section{Results}

Ovariohysterectomy was performed on 13 female dogs, and in four cases (Cases. 15 and 17-19), the owner did not authorize this procedure. The correlation between the cytological and histological results regarding presence of malignancy was observed in 17 cases. However, in two cases (Cases. 4 and 9) the fine-needle aspiration cytology suggested malignant tumor and histological analysis showed a benign process, and in another two cases (Cases. 2 and 20) the fine-needle aspiration cytology suggested benign tumor and histological analysis showed a malignant tumor (Table 1).

Radiographic examinations showed no signs of lung metastases or thorax chest lesions in any dog (Fig. 1 and Fig. 2). CT examination found no alteration in the chest wall or mediastinum (Fig. 3). However, lung metastasis was found in two cases (Cases 15 and 17), and lung atelectatic areas were detected in $28.57 \%$ of the dogs (Cases. 3, 8, 11, 14, 17 and 20). The atelectatic areas were small focal infiltrates and located peripherally (Fig. 4). Case 15 had five solid nodules with regular outline that was well-defined at the left lung parenchyma, thus characteristic for a lung metastasis. The larger nodule measured $0.7 \mathrm{~cm}$ in maximum dimension and was located on the medial portion of the left caudal apical lobe (Fig. 5). Another nodule, measuring $0.4 \mathrm{~cm}$ in maximum dimension, located on the medial portion of the left diaphragmatic lobe presented the signal of a nutrient vessel (Fig. 6). Case 17 had two nodules, one
$0.6 \mathrm{~cm}$ nodule in maximum dimension located on the left posterior cranial apical lobe (Fig. 7) and another measuring $0.9 \mathrm{~cm}$ in maximum dimension with irregular outline, clear-cut limits and surrounded by ground-glass opacity (Fig. 8).

\section{Discussion}

Carcinoma, sarcoma, and malignant mixed tumor are considered the most common malignant mammary tumors [2,3], with the latter occurring most frequently [13]. In the present study, histological examinations showed 13 mammary carcinomas and 6 malignant mixed tumors. However, in two female dogs, histology revealed benign tumors (mammary adenoma and benign mixed tumor) that were found to be malignant by fineneedle aspiration cytology, and other two cases the aspiration cytology suggested malignant tumor and histological analysis showed a benign process. Some authors have discouraged the use of fine-needle aspiration cytology due to its insensitivity in differentiating between malignant and benign mammary tumors [2].

Evaluation of the lungs by imaging studies is necessary in all cases of mammary tumors. Because of the high blood flow and capillary network that provoke slower circulation, the lungs are in addition to the regional lymph nodes the most common sites for metastases $[1,14,15]$. The use of two or three radiographic views to evaluate lung diseases in dogs remains controversial $[6,16]$. The present study employed three views, because
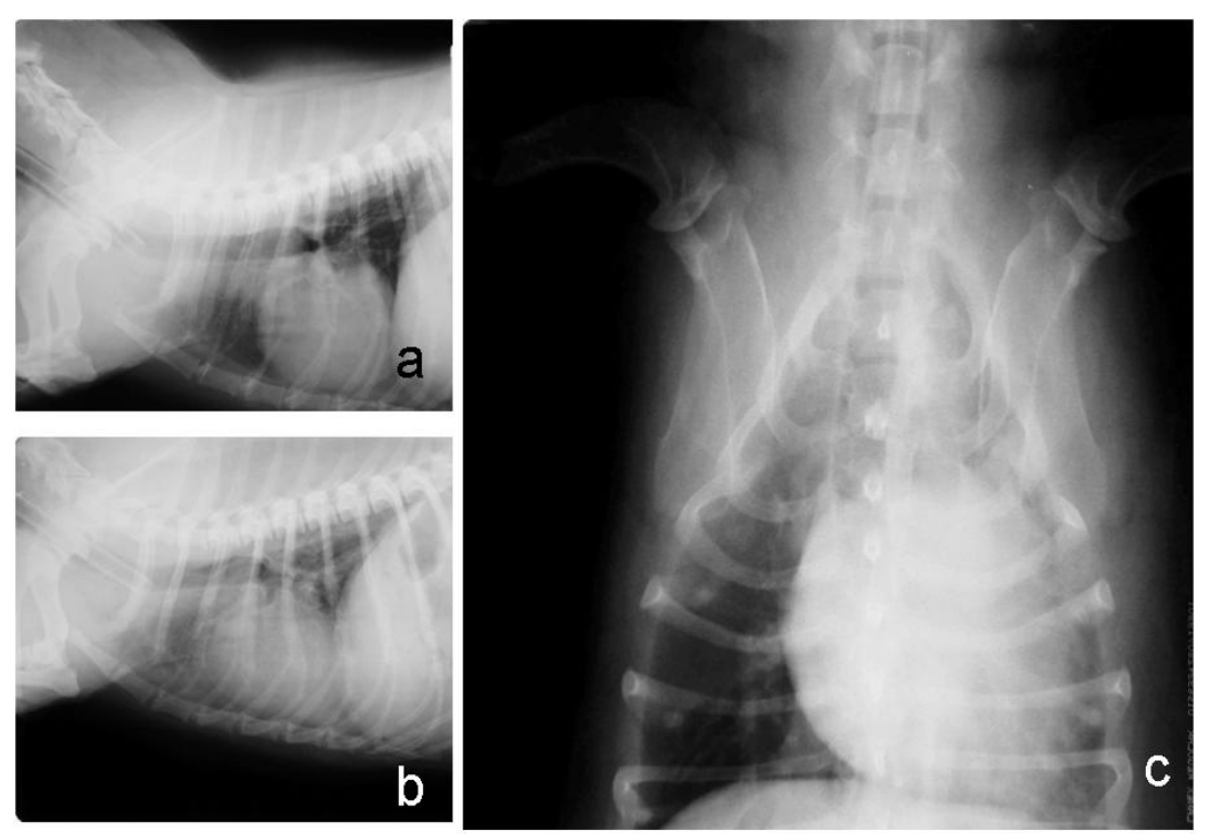

Figure 1 Right lateral (a), left lateral (b), and ventrodorsal (c) radiographic views of the thorax of a female dog (Case 13) with mammary carcinoma. No signs of metastases were observed in the lungs. 


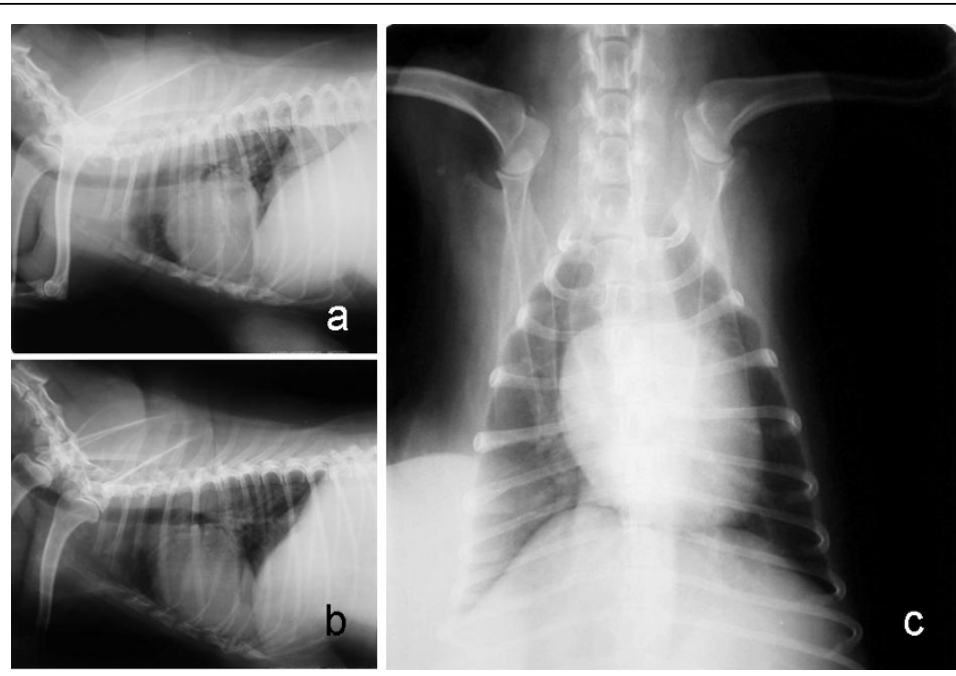

Figure 2 Right lateral (a), left lateral (b), and ventrodorsal (c) radiographic views of the thorax of a female dog (Case 15) with mammary papillary cystadenocarcinoma. No signs of metastases were observed in the lungs.

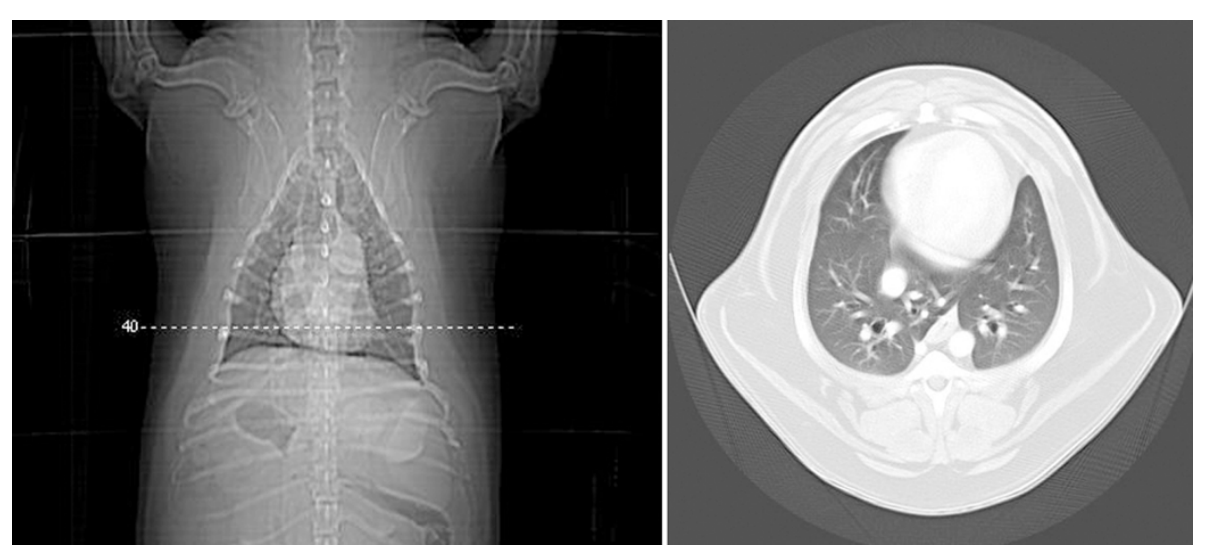

Figure 3 Transverse CT views at the level of the thoracic diaphragmatic lobes of a female dog (Case 13) with mammary carcinoma. No signs of metastases were observed in the lungs.

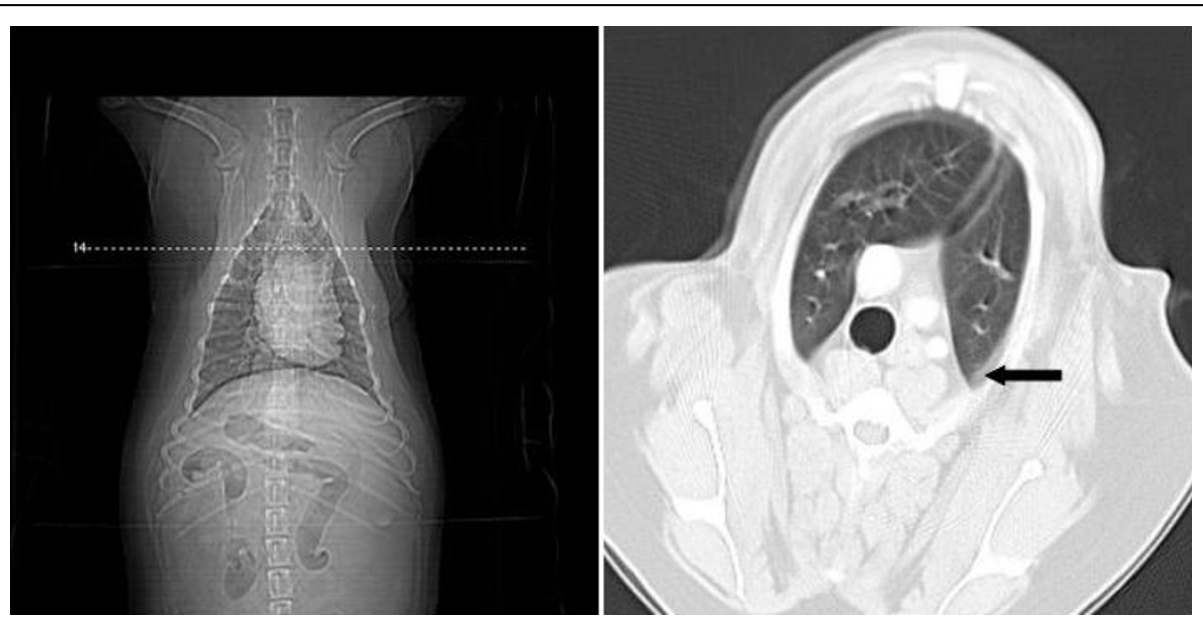

Figure 4 Transverse CT views of the thorax of a female dog (Case 11) showing small area of atelectasis (arrow). 

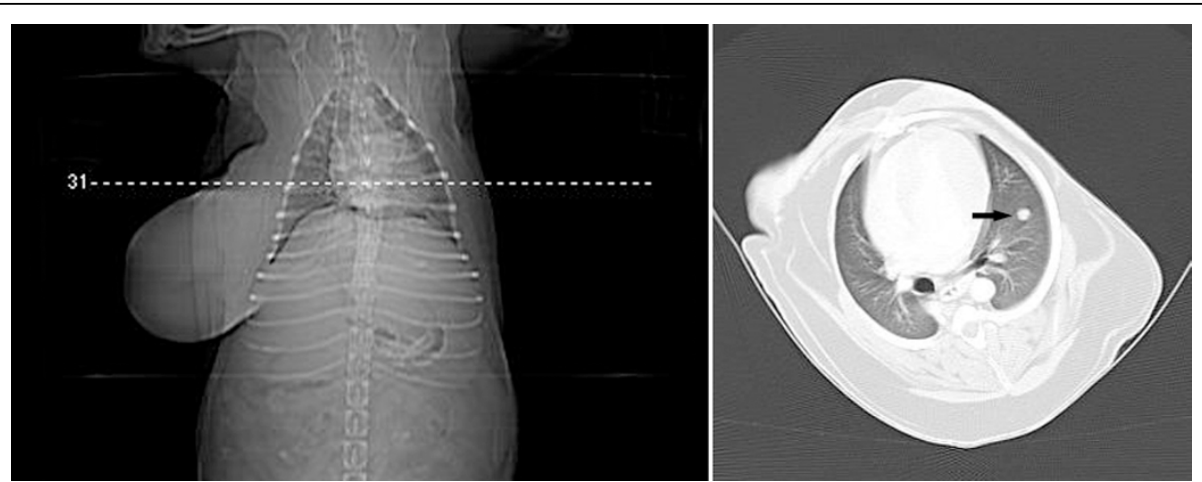

Figure 5 Transverse CT views at the level of the right medial lobe and left apical lobe and thorax of a female dog (Case 15) with mammary papillary cystadenocarcinoma. Observe $0.7 \mathrm{~cm}$ nodule in maximum dimension located on the medial portion of the left caudal apical lobe (arrow). Note tumor present in the right caudal thoracic region.
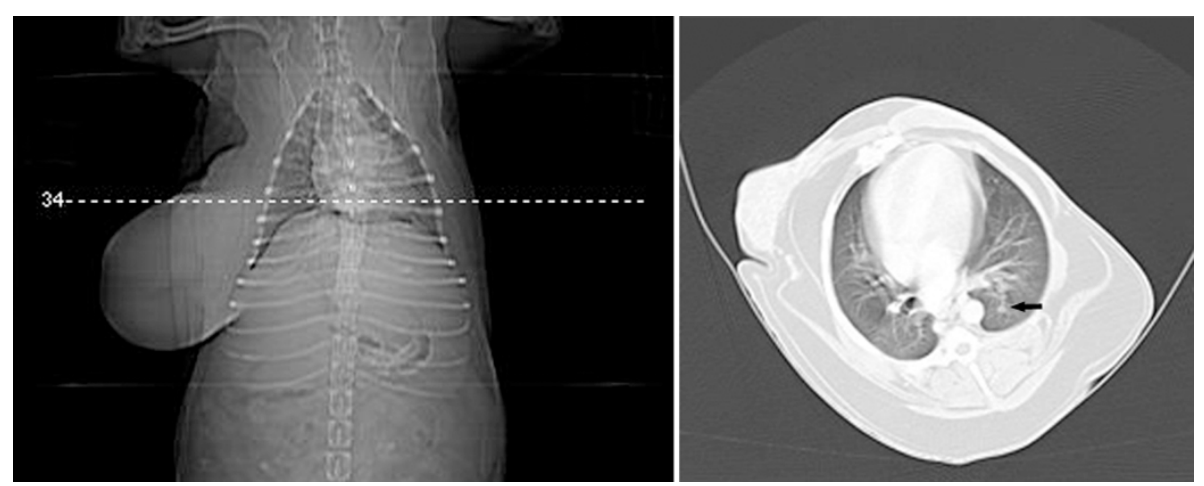

Figure 6 Transverse CT views at the level of the right medial lobe and left diaphragmatic thorax of the female dog (Case 15) with mammary papillary cystadenocarcinoma. Observe $0.4 \mathrm{~cm}$ nodule in maximum dimension located on the medial portion of the left diaphragmatic lobe showing signal of nutrient vessel (arrow).
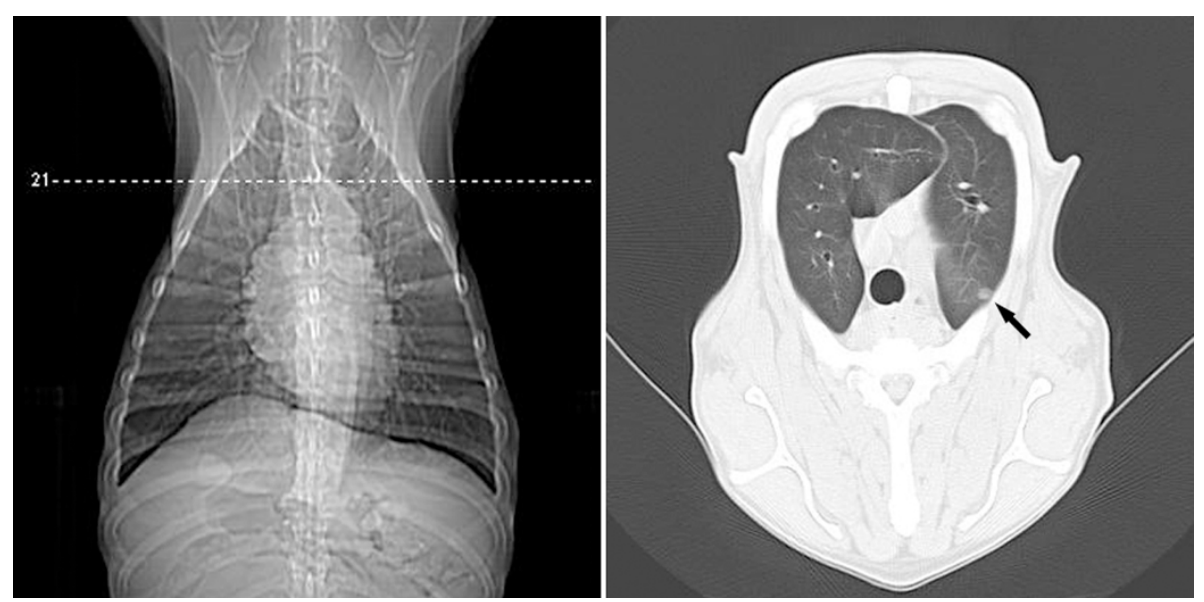

Figure 7 Transverse CT views at the level of the right apical lobe and left thorax of a female dog (Case 17) with malignant mixed mammary tumor. Observe $0.6 \mathrm{~cm}$ nodule in maximum dimension located on the left posterior cranial apical lobe (arrow). 


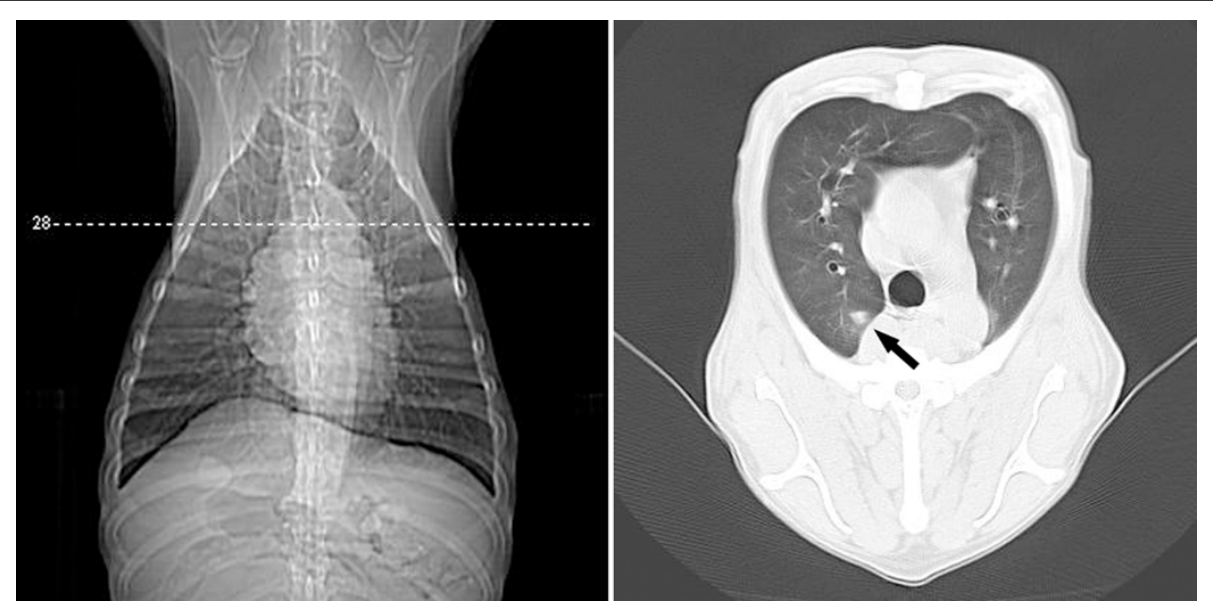

Figure 8 Transverse CT views at the level of the right apical lobe and left thorax of a female dog (Case 17) with malignant mixed mammary tumor. Observe $0.9 \mathrm{~cm}$ nodule in maximum dimension with irregular outline, clear-cut limits and surrounded by ground-glass opacity, located on the posterior portion of the right apical lobe (arrow).

eliminating one radiographic view may influence the diagnosis in $12-15 \%$ of the patients [17]. However, three views were not sufficient to detect the metastases, showing that radiography is less sensitive than CT $[10,11]$. In 18 dogs with pulmonary metastatic neoplasia, only $9 \%$ of the pulmonary nodules detected in CT were observed on radiographs. Besides, pulmonary nodules were identified in a greater number of lung lobes by $\mathrm{CT}$ than radiographs [8].

The dogs were maintained in spontaneous breathing during CT examination due to the type of anesthesia used. However, the images obtained during the apneic period and at the inspiration peak are considered better to minimize the breathing artifact at the tumor location and size $[18,19]$. Some authors have used intermittent positive-pressure ventilation to decrease motion artifacts $[8,20]$. On the other hand, in a study of dogs with metastatic osteosarcoma using spiral CT with a collimation of $5 \mathrm{~mm}$ and pitch of 2, no significant difference was observed between normal resting respiration and holding of breath [21].

Technical modifications may be performed to obtain an optimal lung CT [22]. In a study of two dogs with pulmonary nodules evaluated by helical CT, the suggested protocol included a narrow collimation (3-5 $\mathrm{mm}$ according to the dog's size), pitch of 2 and interval reconstruction of 1 [23]. A similar protocol was used in the present study. CT examinations with intravenous contrast injection were performed to obtain a better definition of the vessels and to differentiate between normal and abnormal vascular structures $[18,24]$. In addition, soft-tissue and lung windows were utilized to enable evaluation of chest wall, and lung parenchyma $[20,18]$.
The dogs were positioned in dorsal recumbency, although some authors suggest that sternal recumbency provides better quality and absence of artifacts [18]. Atelectatic areas were observed by CT in $28.57 \%$ of the cases, but these areas are probably associated with recumbency and gravitational stasis [24] or anesthetic procedure [25], since they are not presented radiographically. Another cause of atelectasia is the transport of the patient under general anesthesia to the CT gantry in lateral recumbency [26]. In a study of dogs with metastatic neoplasia positioned in ventral recumbency, when the initial image showed ventral atelectasis, the CT was repeated with the patient in dorsal recumbency to allow better inflation of these lung regions [8]. The atelectatic areas may show variable CT appearance such as focal interstitial infiltrates, alveolar infiltrates or complete lobar collapse $[19,22]$. In the present study most of these areas were peripheral small focal infiltrates and apparently did not influence the results.

CT allowed detection of metastases in two cases in the present study, thus showing greater precision than the plain radiographic examination that it is considered less sensitive for detection of small lesions [7,8,16,27]. In a retrospective study of 18 dogs with pulmonary metastatic neoplasia, the smallest size to detect pulmonary nodules on CT images was $1 \mathrm{~mm}$, compared with 7-9 $\mathrm{mm}$ on radiographs [8]. However, in a study of four dogs presenting lung metastases of osteosarcoma confirmed by plain thoracic radiographic examination, the spiral CT did not detect 32 metastases measuring $\leq 1 \mathrm{~cm}$ in diameter [10]. Thus, the low number of lung metastases observed in the present study may be underestimated due to the limitation of the imaging examinations used. Probably this fact is associated with the citation 
that most female dogs with malignant tumors without metastases at the moment of the surgery will have died or will have been euthanized on account of problems associated with the tumor within 1 or 2 years [3].

Case no. 17 had five solid nodules with regular outline and was well-defined at the lung parenchyma, which characterizes lung metastases. This standard of lung metastases is compatible with the occurrence of the hematogenous dissemination, which can occur as single, multiple or propagated nodules, with generally random distribution. However, the metastatic nodules are generally circular with regular contour, especially in more aggressive lesions, such as those of sarcomatous origin [28]. Furthermore, the typical metastatic carcinoma usually occurs as numerous small circular lesions [29].

Case 15 had two pulmonary nodules, one of which presented an irregular outline, clear-cut limits and was surrounded by ground-glass opacity. The metastatic nodules may have irregular or lobular contour [28].

\section{Conclusions}

In this study population, spiral CT showed higher sensitivity than chest radiographies to detect lung metastasis; this indicates that CT should be performed on all female dogs with malignant mammary tumors.

\section{Acknowledgements}

We are grateful to Fapesp for the spiral Scanner and to Capes for the fellowship.

\section{Author details \\ 'São Paulo State University (Unesp), Department of Veterinary Surgery and Anesthesiology, School of Veterinary Medicine and Animal Science, Botucatu, SP, Brazil. 'Unesp, Department of Animal Reproduction and Radiology, School of Veterinary Medicine and Animal Science, Botucatu, SP, Brazil. ${ }^{3}$ Unesp, Department of Tropical Diseases and Diagnostic Imaging, Botucatu Medical School, Botucatu, SP, Brazil. ${ }^{4}$ Unesp, Department of Veterinary Clinical Sciences, School of Veterinary Medicine and Animal Science, Botucatu, SP, Brazil.}

\section{Authors' contributions}

$\mathrm{CCO}, \mathrm{SCR}$ and $\mathrm{KH}$ participated in the study design, surgical procedures, and drafted the manuscript. LCV, SMR and DPD interpreted the radiographic and $C T$ examinations. TG performed the anesthesia procedures. RLA performed the microscopic examinations. All authors read and approved the final manuscript.

\section{Competing interests}

The authors declare that they have no competing interests.

Received: 18 June 2009 Accepted: 9 March 2010

Published: 9 March 2010

\section{References}

1. Harvey JH: Mammary glands. Current techniques in small animal surgery Baltimore: Williams \& WilkinsBojrab MJ, Ellison GW, Slocum B 1998, 579-584.

2. Robbins M: Reproductive oncology. Textbook of small animal surgery Philadelphia: SaundersSlatter D 2003, 2437-2443.

3. Hedlund CS: Mammary neoplasia. Small animal surgery St. Louis: Mosby ElsevierFossum TW 2007, 729-735.
4. Sorenmo KU, Shofer FS, Goldschmidt MH: Effect of spaying and timing of spaying on survival of dogs with mammary carcinoma. J Vet Intern Med 2000, 14:266-270.

5. Chang SC, Chang CC, Chang TJ, Wong ML: Prognostic factors associated with survival two years after surgery in dogs with malignant mammary tumours: 79 cases (1998-2002). J Am Vet Med Assoc 2005, 227:1625-1629.

6. Barthez PY, Hornof WJ, Theon AP, Craychee TJ, Morgan JP: Receiver operating characteristic curve analysis of the performance of various radiographic protocols when screening dogs for pulmonary metastases. J Am Vet Med Assoc 1994, 204:237-240.

7. Baumann D, Hauser B, Hubler M, Fluckiger M: Signs of metastatic disease on thoracic radiographs of dogs suffering from mammary gland tumours: a retrospective study (1990-1998). Schweiz Arch Tierheilkd 2004, 146:431-435.

8. Nemanic S, London CA, Wisner ER: Comparison of thoracic radiographs and single breath-hold helical CT for detection of pulmonary nodules in dogs with metastatic neoplasia. J Vet Intern Med 2006, 20:508-515.

9. Schafer JF, Vollmar J, Schick F, Seemann MD, Kamm P, Erdtmann B, Claussen CD: Detection of pulmonary nodules with breath-hold magnetic resonance imaging in comparison with computed tomography. Rofo 2005, 177:41-49.

10. Waters DJ, Coakley FV, Cohen MD, Davis MM, Karmazyn B, Gonin R, Hanna MP, Knapp DW, Heifetz SA: The detection of pulmonary metastases by helical CT: a clinicopathologic study in dogs. J Comput Assist Tomogr 1998, 22:235-240.

11. Coakley FV, Cohen MD, Johnson MS, Gonin R, Hanna MP: Maximum intensity projection images in the detection of simulated pulmonary nodules by spiral CT. Br J Radiol 1998, 71:135-140.

12. Johnston SD: Reproductive system. Textbook of Small Animal Surgery San Diego: SaundersSlatter D 2003, 2566-2583.

13. Jones $\mathrm{CJ}$, Hunt RD, King NW: Genital system. Veterinary Pathology Indianapolis: Wiley-BlackwellJones TC, Hunt RD, King NW 1997, 1169-1244.

14. Cotran RS, Kumar V, Collins T: Neoplasia. Pathologic Basis of Disease Philadelphia: SaundresRobbins SL 1989, 233-295.

15. Rubin E, Farber JL: Neoplasia. Pathology Baltimore: Lippincott Williams \& WilkinsRubin E, Farber JL 1999, 152-209.

16. Holt D, Van Winkle T, Schelling C, Prymak C: Correlation between thoracic radiographs and postmortem findings in dogs with hemangiosarcoma: 77 cases (1984-1989). J Am Vet Med Assoc 1992, 200:1535-1539.

17. Ober CP, Barber D: Comparison of two -vs. three-view thoracic radiographic studies on conspicuity of structured interstitial patterns in dogs. Vet Radiol Ultrasound 2006, 47:542-545.

18. Cardoso L, Gil F, Ramírez G, Teixeira MA, Agut A, Rivero MA, Arencibia A, Vásquez JM: Computed tomography (CT) of the lungs of the dog using a helical CT scanner, intravenous iodine contrast medium and different CT windows. Anat Histo Embryol 2007, 36:328-331.

19. Morandi F, Matton JS, Lakritz J, Turki JR, Wisner ER: Correlation of helical and incremental high-resolution thin-section computed tomographic imaging with histomorphometric quantitative evaluation of lungs in dogs. AJVR 2003, 64(Suppl 7):935-944.

20. Coakley FV, Cohen MD, Waters DJ, Davis MM, Karmazyn B, Gonin R, Hanna MP: Detection of pulmonary metastases with pathological correlation: effect of breathing on the accuracy of spiral CT. Pediatr Radiol 1997, 27:576-579.

21. Rycle LM, Gielen IM, Simoens PJ, Van Bree H: Computed tomography and cross-sectional anatomy of the thorax in clinically normal dogs. Am J Vet Res 2005, 66:512-524.

22. Webb WR, Müller NL, Naidich DP: HRCT findings of lung disease. Highresolution CT of the lung Philadelphia: Lippincott-RavenWebb WR 1996, 41-108.

23. Joly H, D'Anjou M, Alexander K, Beauchamp G: Comparison of single-slice computed tomography protocols for detection of pulmonary nodules in dogs. Veterinary Radiology \& Ultrasound 2009, 50(Suppl 3):279-284.

24. Widmer WR: Alternate imaging for diagnosis of cancer. Cancer in dogs and cats: medical and cirurgical management Baltimore: Teton NewmediaMorrison WB 2002, 177-204.

25. Warner DO, Warner MA, Ritman EL: Atelectasis and chest wall shape during anesthesia. Anestediology 1996, 85(Suppl 1):49-59.

26. Johnson EG, Wisner ER: Advances in respiratory imaging. Vet Clin Small Anim 2007, 37:879-900. 
27. Ketai L, Malby M, Jordan K, Meholic A, Locken J: Small nodules detected on chest radiography: does size predict calcification?. Chest 2000, 118:610-614.

28. Oliveira AC: Metastases pulmonares. Doenças Pulmonares Rio de Janeiro: Ganabara KooganTarantino AB 2002, 717-724.

29. Farrow CS: Lung neoplasm. Veterinary Diagnostic Imaging: the dog and the cat New York: MosbyFarrow CS 2003, 425-437.

doi:10.1186/1751-0147-52-20

Cite this article as: Otoni et al: Survey radiography and computerized tomography imaging of the thorax in female dogs with mammary tumors. Acta Veterinaria Scandinavica 2010 52:20.

Submit your next manuscript to BioMed Central and take full advantage of:

- Convenient online submission

- Thorough peer review

- No space constraints or color figure charges

- Immediate publication on acceptance

- Inclusion in PubMed, CAS, Scopus and Google Scholar

- Research which is freely available for redistribution

Submit your manuscript at www.biomedcentral.com/submit
C Biomed Central 